\title{
Imaging the mitral valve: a primer for the interventional surgeon
}

\author{
Aditya Sengupta ${ }^{1}$, Sophia L. Alexis ${ }^{1}$, Syed Zaid ${ }^{2}$, Gilbert H. L. Tang ${ }^{1}$, Stamatios Lerakis ${ }^{3}$, \\ Randolph P. Martin ${ }^{4}$
}

${ }^{1}$ Department of Cardiovascular Surgery, Mount Sinai Hospital, New York, NY, USA; ${ }^{2}$ Department of Cardiology, Heart and Vascular Institute, Westchester Medical Center, Valhalla, New York, NY, USA; ${ }^{3}$ Division of Cardiology, Mount Sinai Hospital, New York, NY, USA; ${ }^{4}$ Division of Cardiology, Department of Medicine, Emory University School of Medicine, Atlanta, GA, USA

Correspondence to: Randolph P. Martin, MD, FASE, FACC, FESC. Emeritus Professor of Cardiology, Division of Cardiology, Department of Medicine, Emory University School of Medicine, 3360 Nancy Creek Rd NW, Atlanta, GA 30327, USA. Email: randolphmmd@gmail.com.

\begin{abstract}
Transcatheter mitral valve interventions (TMVI) have evolved over the past decade as alternatives to open surgical repair for the therapeutic management of patients with severe mitral regurgitation (MR). Concurrent with the development of these technologies, quality multi-modality cardiac imaging has become essential in patient selection and procedural guidance. The former involves assessments of the pathophysiologic mechanisms of regurgitation, valvular anatomy and morphology, as well as objective quantification of the severity of MR. Both transthoracic and transesophageal echocardiography (TEE) are crucial and serve as the gateway to diagnosis and management of mitral valvular disease. Along with multi-detector computed tomography (CT) and cardiac magnetic resonance imaging (CMR), echocardiography plays an important role for preprocedural planning and evaluation of the spatial relationships of the mitral valvular complex with the coronary sinus, circumflex coronary artery and left ventricular (LV) outflow tract. Procedures that target mitral leaflets (e.g., MitraClip, PASCAL) or annulus (e.g., Cardioband, Carillon), or provide chordal (e.g., NeoChord, Harpoon) or valvular replacement, tend to be guided by TEE and assisted by fluoroscopy. As newer devices become available and outcomes of TMVI improve, cardiac imaging will undoubtedly continue to play an essential role in the success of percutaneous mitral valve repair (MVr) and replacement. The interventional surgeon of the future must therefore have a thorough understanding of the various imaging modalities while synthesizing and integrating novel concepts (e.g., neo-LV outflow tract) as applicable to assessing valvular function and pathology.
\end{abstract}

Keywords: Mitral regurgitation (MR); multimodality cardiac imaging; interventional echocardiography; computed tomography (CT); transcatheter mitral valve replacement (TMVR)

Submitted Apr 09, 2020. Accepted for publication Oct 27, 2020.

doi: $10.21037 /$ acs-2020-mv-16

View this article at: http://dx.doi.org/10.21037/acs-2020-mv-16

\section{Introduction}

Moderate or greater mitral regurgitation (MR) is the most prevalent valvular heart disease among adults in the United States (1). Although surgical mitral valve repair $(\mathrm{MVr})$ remains the standard of care for severe, symptomatic MR, the increasing number of patients who present with contraindications to surgery or who are at high operative risk poses an ongoing therapeutic challenge (2). In turn, percutaneous technologies have evolved over the past decade as feasible and safe alternatives to open-heart surgery in select patients. Concurrently, multimodality imaging has become crucial in both patient selection and procedural guidance (3). The former is based on noninvasive imaging techniques, such as transthoracic echocardiography (TTE), transesophageal echocardiography (TEE) and multidetector row computed tomography (MDCT), that allow thorough assessments of valve morphology, quantification of regurgitation, optimization of timing of intervention, 

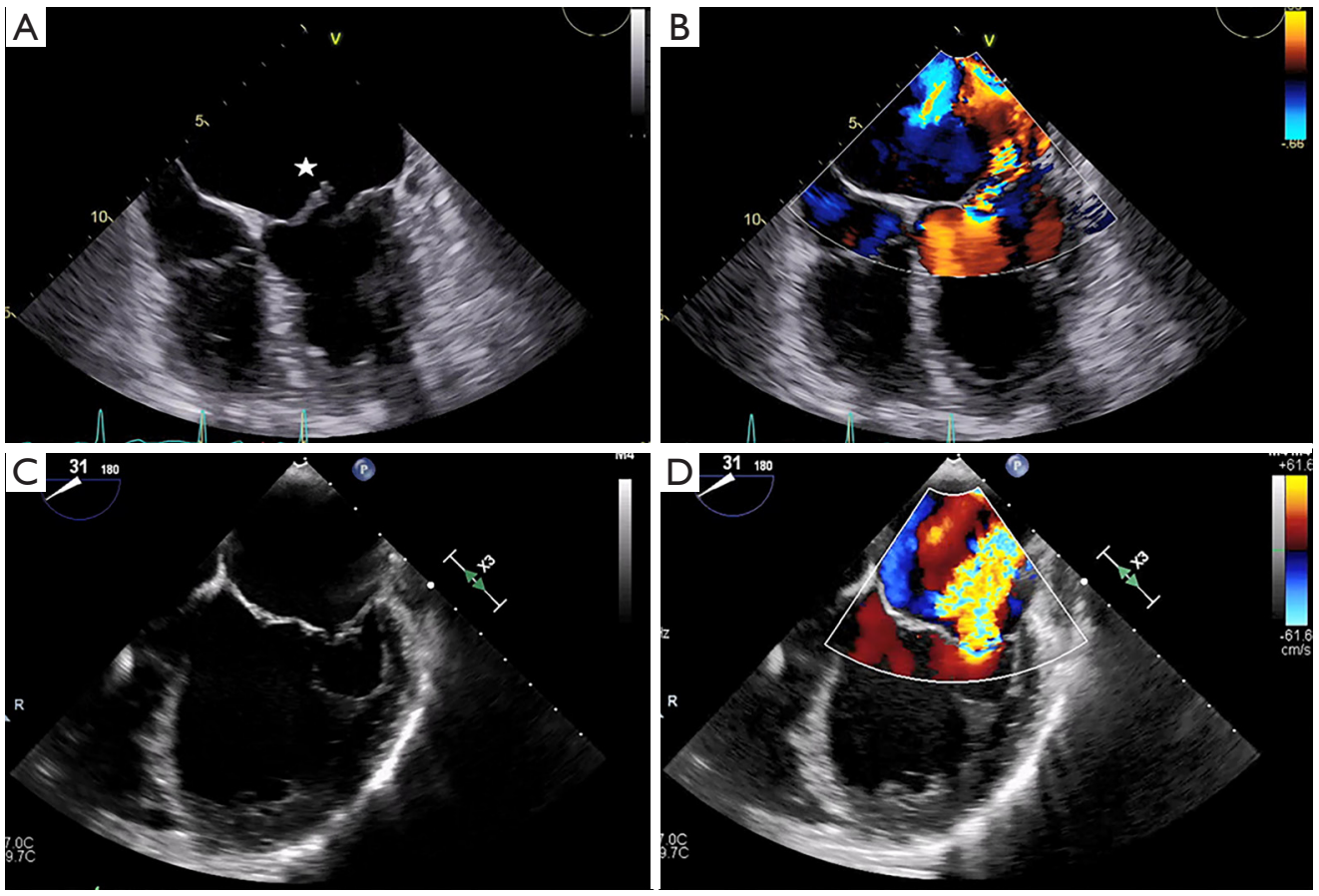

Figure 1 Pathophysiology of mitral regurgitation. Ascertaining the pathophysiologic mechanism(s) of MR is a key step in evaluating patients for TMVI. (A,B) Primary MR occurs as a result of degenerative disease affecting one or more components of the mitral valvular complex. A large A2-A3 flail anterior leaflet (star) with torrential MR is demonstrated. (C,D) In contrast, secondary MR is a consequence of left ventricular dilatation and remodeling, which leads to leaflet tethering and failure of coaptation. Echocardiography here shows severe functional MR in a patient with an ejection fraction of $15 \%$. A2, middle segment of the anterior mitral leaflet; A3, posterior segment of the anterior mitral leaflet; MR, mitral regurgitation; TMVI, transcatheter mitral valve intervention.

device selection and procedural planning. Similarly, fluoroscopy and TEE provide essential intraoperative imaging guidance for proper device deployment (4). Here, we provide a comprehensive review of the various cardiac imaging modalities in the context of the transcatheter mitral valve interventions (TMVI) that are currently available.

\section{Patient selection}

Cardiac imaging underlies proper patient selection for TMVI and guides clinicians on the optimal timing of intervention. The process starts with a thorough understanding of the various pathophysiologic mechanisms of MR, followed by anatomic evaluation of the mitral valve (MV) and grading of MR severity. Two-dimensional TTE (TTE-2D), TEE and stress echocardiography have become invaluable in not only diagnosing and managing $\mathrm{MV}$ disease, but also identifying patients that may benefit from earlier intervention.

\section{Pathophysiologic mechanisms of MR}

Once MR has been detected, patient evaluation often begins with an assessment of the mechanism of regurgitation. Consensus statements from numerous societal guidelines generally categorize MR as either primary or secondary $(5,6)$. Primary (also called degenerative or organic) MR (Carpentier type II) occurs as a result of an intrinsic degenerative process of the MV leaflets in patients with MV prolapse (Figure 1A,B). In contrast, secondary (or functional) MR (Carpentier type IIIB) occurs in patients with valves that have been stretched by tethering or left ventricular (LV) dilatation (Figure 1C,D). The valve remains anatomically normal, but apical and lateral distraction of the papillary muscles from ventricular dilatation leads to leaflet tethering and central regurgitation due to failure of coaptation $(5,7)$.

Both MV dysfunction and the etiology of MR are characterized mainly by TTE and TEE. These modalities allow morphological analyses of the MV and its subvalvular 

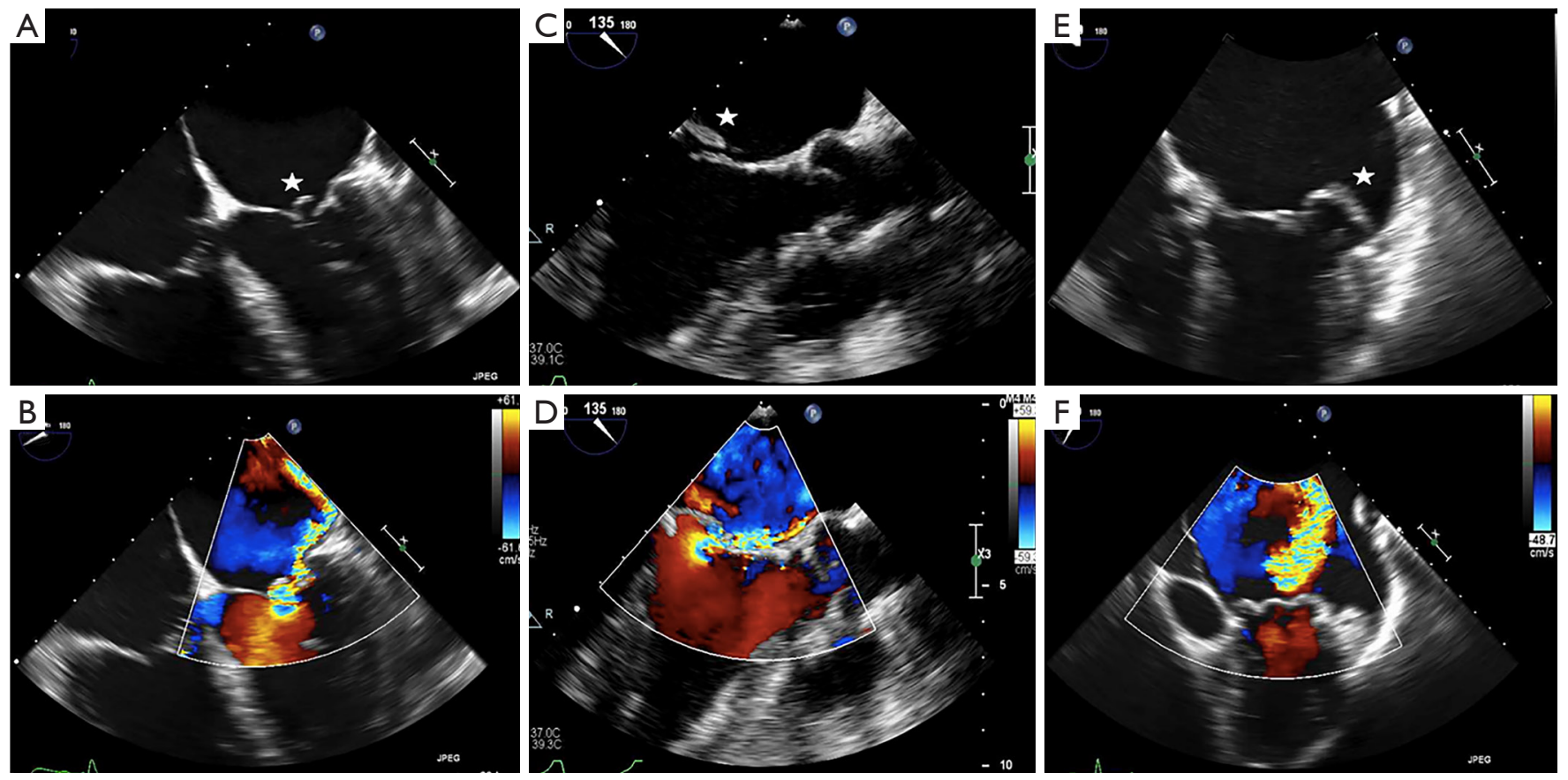

Figure 2 Morphological analysis of the mitral valve in primary mitral regurgitation. Preprocedural TEE is often useful in distinguishing between Barlow's disease and fibroelastic deficiency in patients with primary MR. (A,B) The four-chamber view shows a flail A1 segment (star) of the anterior leaflet and severe MR. (C,D) A flail P2 scallop (star) with severe MR is seen on the AV long-axis view. (E,F) TEE here shows torrential MR due to lateral commissural prolapse extending to the P1 and P2 segments (star), with billowing well into the left atrium. A1, anterior segment of the anterior mitral leaflet; $\mathrm{AV}$, aortic valve; $\mathrm{MR}$, mitral regurgitation; $\mathrm{P} 1$, anterior segment of the posterior mitral leaflet; $\mathrm{P} 2$, middle segment of the posterior mitral leaflet; TEE, transesophageal echocardiography.

apparatus. Three-dimensional TEE (TEE-3D) mitral anatomy correlates well with findings during open-heart surgery (8). Echocardiography also permits segmental analysis of the leaflet scallops and commissures, thus allowing the interventional surgeon to precisely locate the focus of valvular dysfunction. This is particularly true in cases of primary MR, where echocardiography can make the distinction between Barlow's disease (e.g., multisegment redundancy, billowing and thickened leaflet tissue) and fibroelastic deficiency (e.g., chordal rupture involving a single scallop) (Figure 2) (4).

\section{Valvular anatomy \& imaging considerations}

The MV complex consists of the anterior and posterior leaflets, a fibrous annulus with varying degrees of continuity and integrity and the subvalvular apparatus, consisting of the chordae tendineae and papillary muscles (9). Key TTE views that identify anatomic and functional abnormalities of one or more of these valvular components include: (I) the $2 \mathrm{D}$ long-axis view that runs through the middle portion of the anterior leaflet (A2) and the middle scallop of the posterior leaflet (P2), (II) the short-axis view that allows imaging of both leaflets and the commissures and (III) the four-chamber view that cuts through the leaflets at different locations (Figure 3). Similarly, important TEE views include: (I) the cross-commissural view (or transthoracic apical two-chamber view) that identifies A2 along with the lateral (P1) and medial (P3) scallops of the posterior leaflet and (II) the 3D en face that provides optimal views of the line of coaptation (10).

In addition to echocardiography, MDCT is increasingly becoming an important modality to characterize $\mathrm{MV}$ anatomy and evaluate the feasibility of TMVI (Figure 4). Electrocardiogram-gated MDCT allows retrospective acquisition of images throughout the cardiac cycle. These are then reconstructed at $5 \%$ or $10 \%$ of the RR interval in multiple reformation planes (4). MV parameters that need to be assessed with MDCT for successful TMVI are being actively investigated and empirically include mitral annular 

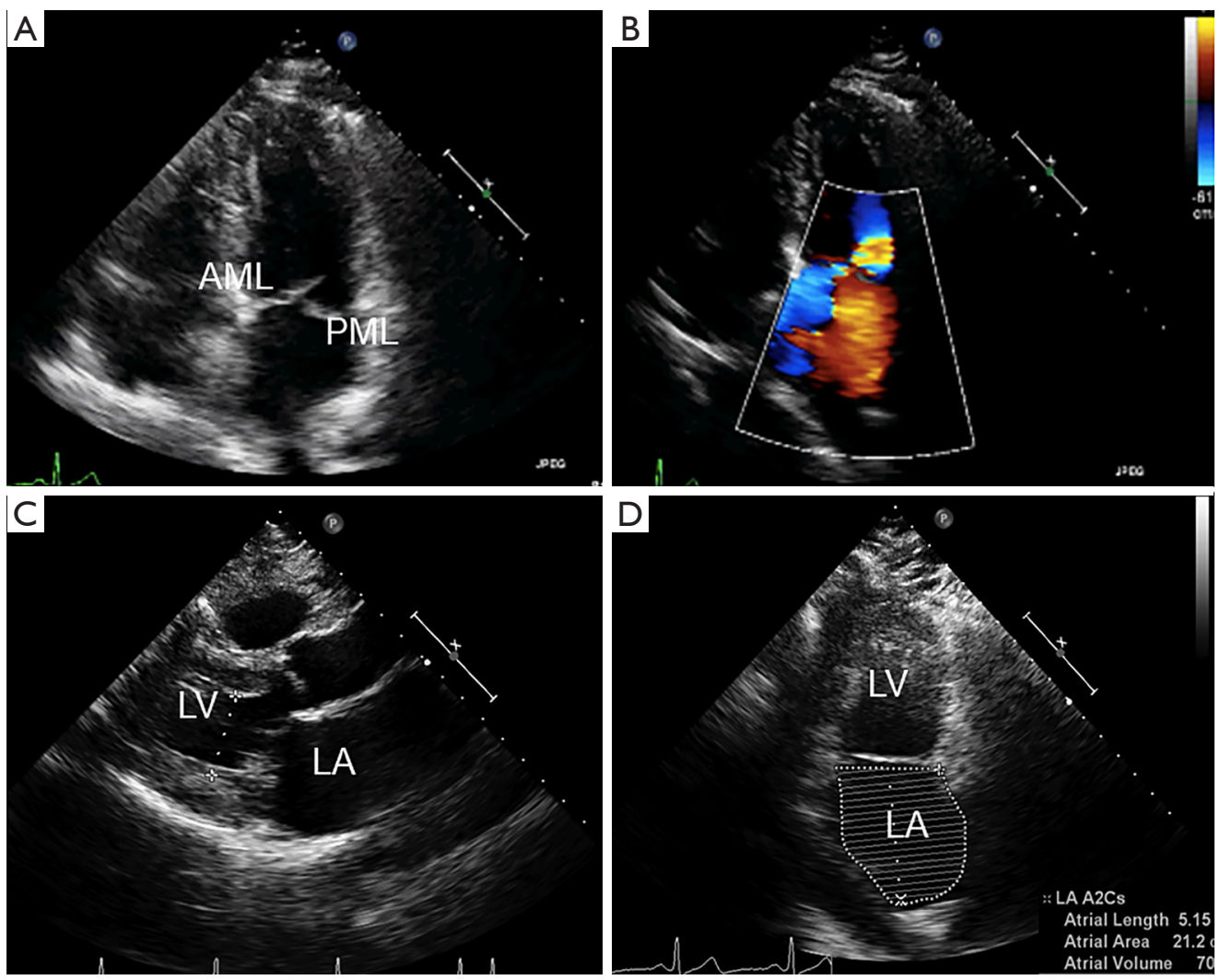

Figure 3 Preprocedural anatomic assessment with transthoracic echocardiography. TTE remains the cornerstone of anatomic evaluation in patients with mitral valve disease. (A) The apical four-chamber view visualizes both mitral leaflets and their coaptation, which should be at the level of the mitral annulus. Here, we see PML prolapse. (B) Color Doppler imaging reveals moderate to severe MR with an anteriorly directed jet. (C) The parasternal long-axis view allows for assessment of leaflet thickness and motion during the cardiac cycle. The left ventricular end-systolic dimension here is measured to be $2.8 \mathrm{~cm}$. (D) The apical two-chamber view is essential for evaluating the different segments of the mitral leaflets and the severity of MR. Here, the LA volume index was measured at $60 \mathrm{~mL} / \mathrm{m}^{2}$. AML, anterior mitral leaflet; LA, left atrium; MR, mitral regurgitation; PML, posterior mitral leaflet; TTE, transthoracic echocardiography.

dimensions (perimeter and inter-trigonal, inter-commissural and septolateral distances), mitral annular calcification (MAC), leaflet length, distance between papillary muscles, LV outflow tract (LVOT) area and the course of the coronary sinus and circumflex coronary artery relative to the annular plane $(4,11)$.

MDCT also has a role in assessing mitral annular geometry and the risk of LVOT obstruction (LVOTO) following transcatheter mitral valve replacement (TMVR) (Figure 5). Numerous studies have postulated that this risk arises from the creation of a neo-LVOT as a result of protrusion of the TMVR device and anterior mitral leaflet into the native LVOT (12). A recent report by Yoon et al. demonstrated that MDCT-based estimations of neo-LVOT area $\leq 1.7 \mathrm{~cm}^{2}$ predicted LVOTO with a sensitivity of $96 \%$ and specificity of $92 \%$ following TMVR, thus cementing its role in analyzing the spatial relationship between the left ventricle and its outflow tract during transcatheter interventions (13).

\section{Assessment of MR severity}

The American College of Cardiology/American Heart Association (ACC/AHA) recommends TTE and TEE as the first-line imaging modalities for MR assessment (6). In concordance with this, baseline MR should be characterized using multi-parametric and multi-modality imaging that allows for both qualitative and quantitative evaluation of MR severity (Table 1) $(5,6,10)$.

Color Doppler imaging is the most commonly used 

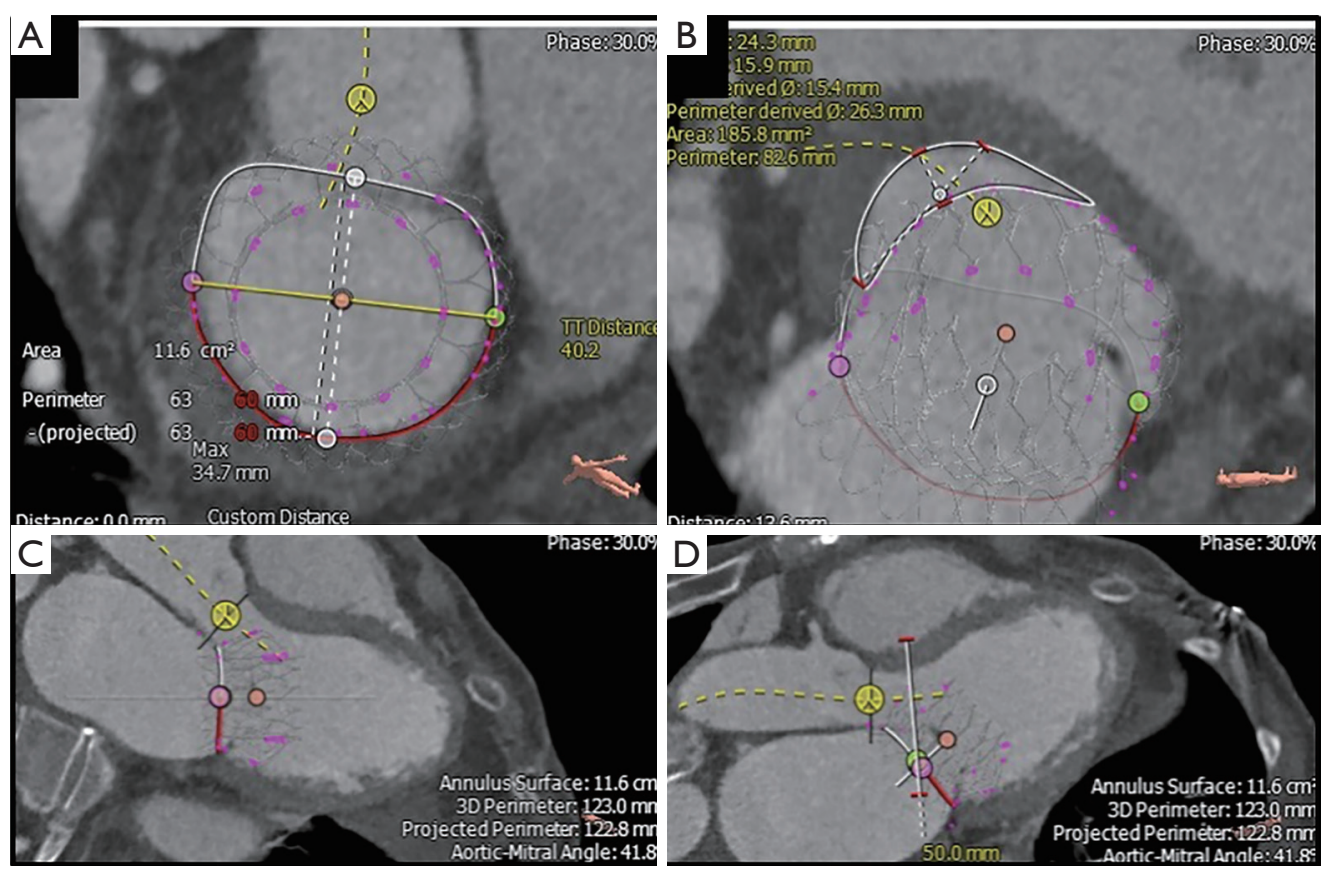

Figure 4 Preprocedural multidetector computed tomography analysis. Using the 3 Mensio Valves software, MDCT reconstruction can be used for preprocedural evaluation of valvular anatomy to determine the feasibility of TMVR. (A) First, annular dimensions (intercommissural maximal diameter and anterior-posterior diameter) are determined. (B) Next, the neo-LVOT area is measured by overlaying a virtual TMVR device in the appropriate position $\left(1.86 \mathrm{~cm}^{2}\right.$ in this case). (C) The virtually-placed TMVR device across the mitral annulus is used to assess LVOT clearance in a 3-chamber view. (D) The white line cutting across the LVOT and virtual TMVR device demarcates the cut-plane used to determine the neo-LVOT area and assess the risk of LVOT obstruction. MDCT, multidetector computed tomography; LVOT, left ventricular outflow tract; TMVR, transcatheter mitral valve replacement.

qualitative technique of assessing MR. Large, eccentric jets that adhere, swirl and reach the posterior wall of the left atrium usually signify severe MR. Furthermore, the presence of a large proximal flow convergence, a dense or triangular continuous wave Doppler (CWD) signal of the regurgitant jet and vena contracta $(\mathrm{VC})$ width $\geq 0.7 \mathrm{~cm}$ suggests significant MR. Although useful, color Doppler measurements are highly variable as they are dependent on technical and hemodynamic parameters (14). MR severity can also be objectively assessed using quantitative variables, such as regurgitant volume (RVol), regurgitant fraction $(\mathrm{RF})$ and effective regurgitant orifice area (EROA). These measurements can be performed using the proximal isovelocity surface area (PISA) method (not recommended for eccentric jets, multiple jets or complex and elliptical regurgitant orifices), quantitative Doppler analysis or $3 \mathrm{D}$ color Doppler VC planimetry (15).

In recent years, cardiac magnetic resonance imaging (CMR) has emerged as an adjunct to primary echocardiographic analysis (16). The American Society of Echocardiography (ASE) recommends the use of CMR when echocardiography is felt to be unsatisfactory or when discrepancies exist between MR severity and clinical findings. Reports of comparative studies suggest concordance rates of $50 \%$ to $90 \%$ between the two modalities in defining the severity of MR using semiquantitative parameters (17). Unlike echocardiography, CMR is less influenced by geometric assumptions, jet direction and the presence of multiple regurgitant jets. However, while it provides reliable measurements of RVol and RF, the feasibility of CMR for assessing the etiology of MR and predicting the success of TMVI have yet to be determined (10).

\section{Preprocedural planning}

Each of the various TMVI technologies targets a particular structure of the mitral valvular complex, and are thus generally categorized by the mechanism of MR reduction. As discussed below, multi-modality cardiac imaging plays an 

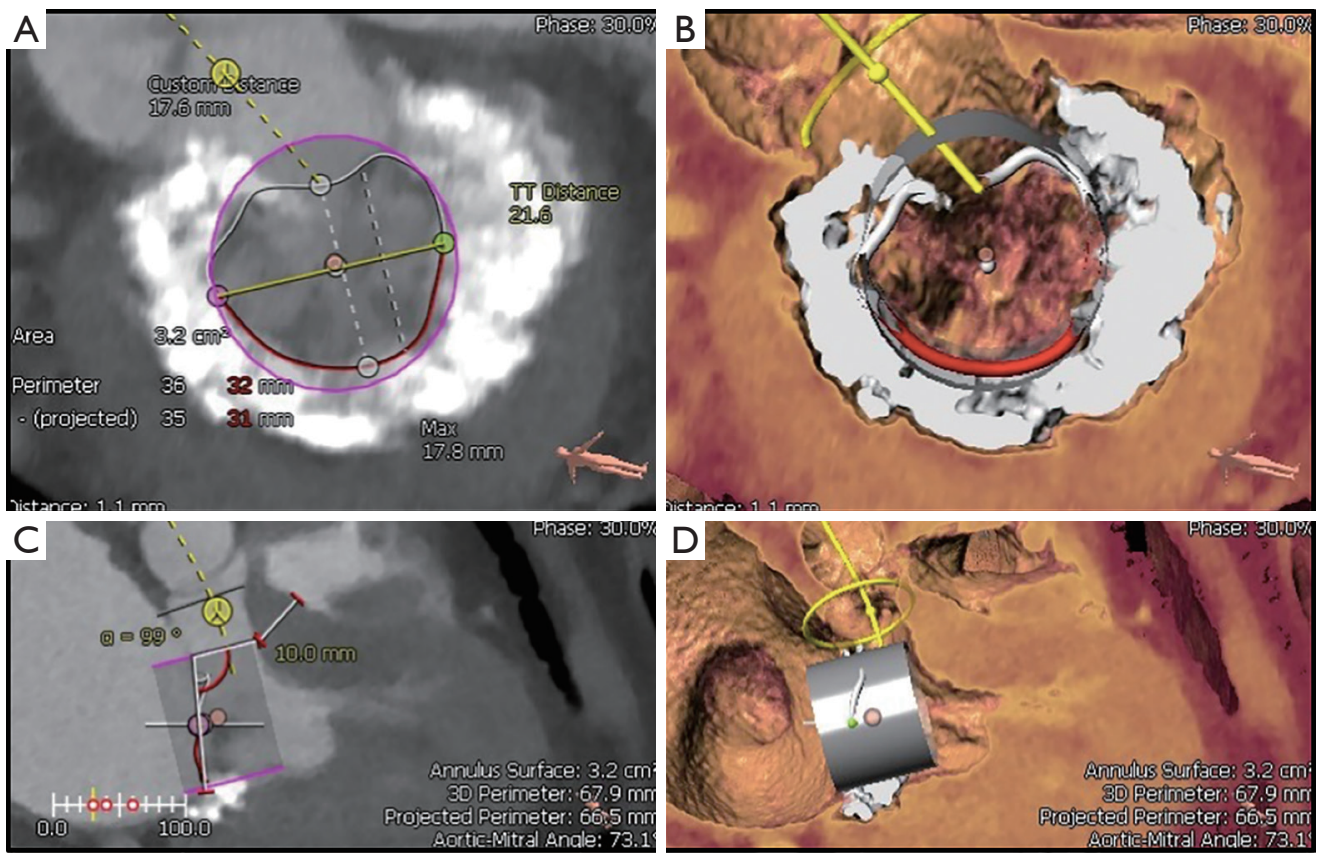

Figure 5 Assessing risk of LVOTO with MDCT analysis in severe MAC. Preoperative MDCT and the 3 Mensio Valves software can be used to predict the risk of LVOTO. (A) Severe, horseshoe MAC is seen encroaching the annulus. Annular dimensions suggest that TMVR with a $23 \mathrm{~mm}$ Edwards SAPIEN 3 valve (purple circle) may be feasible. (B) 3D reconstruction of the en face view. (C) The 3-chamber long-axis view allows virtual placement of the SAPIEN 3 valve (purple rectangle) across the annulus. Complete LVOTO is seen here, thus precluding the use of TMVR in this patient. (D) 3D reconstruction of the 3-chamber view. LVOTO, left ventricular outflow tract obstruction; MAC, mitral annular calcification; MDCT, multidetector computed tomography; TMVR, transcatheter mitral valve replacement; $3 \mathrm{D}$, three-dimensional.

essential role in determining the most appropriate TMVI strategy as well as in preprocedural planning.

\section{TMVI targeting MV leaflets}

Devices that act on the mitral leaflets include MitraClip (Abbott Structural Heart, Santa Clara, CA), PASCAL (Edwards Lifesciences Inc., Irvine, CA), NeoChord DS 1000 (NeoChord Inc., St. Louis Park, MN) and the Harpoon repair system (Edwards Lifesciences Inc., Irvine, CA).

\section{MitraClip \& PASCAL}

The MitraClip percutaneous edge-to-edge repair system has gained United States Food and Drug Administration (FDA) approval for both degenerative and functional MR and is the most commonly used TMVI technology (15). Anatomic inclusion criteria in the original Endovascular Valve Edge-to-Edge Repair Study (EVEREST) trial, as defined by echocardiography, included non-rheumatic valve morphology, mitral valve area $(\mathrm{MVA}) \geq 4 \mathrm{~cm}^{2}$, flail gap $\leq 10 \mathrm{~mm}$, flail width $\leq 15 \mathrm{~mm}$, coaptation depth $\leq 11 \mathrm{~mm}$, coaptation length $\geq 2 \mathrm{~mm}$ and central pathology at the A2P2 interface (18). Since 2009, however, clinical experience has demonstrated the success of TMVI with MitraClip in patients who do not strictly meet the aforementioned criteria, and there currently exist few absolute anatomic contraindications to its use (19). Relative echocardiographic contraindications include calcification at the grasping zone site, MVA $<3.5 \mathrm{~cm}^{2}$, baseline mean mitral gradient $>4 \mathrm{mmHg}$, leaflet grasping length $<7 \mathrm{~mm}$ and Carpentier IIIA physiology $(19,20)$.

TEE is also used for anatomic screening and procedural planning (15). For instance, the biplane commissural view interrogates the $\mathrm{MV}$ across the commissures and can be useful for analyzing valvular anatomy at the grasping location and planning the "grasping view" (Figure 6). Long and redundant leaflets, along with larger coaptation gaps, may necessitate use of the XTR system as opposed to the NTR device (21).

The PASCAL repair system can be considered as an 


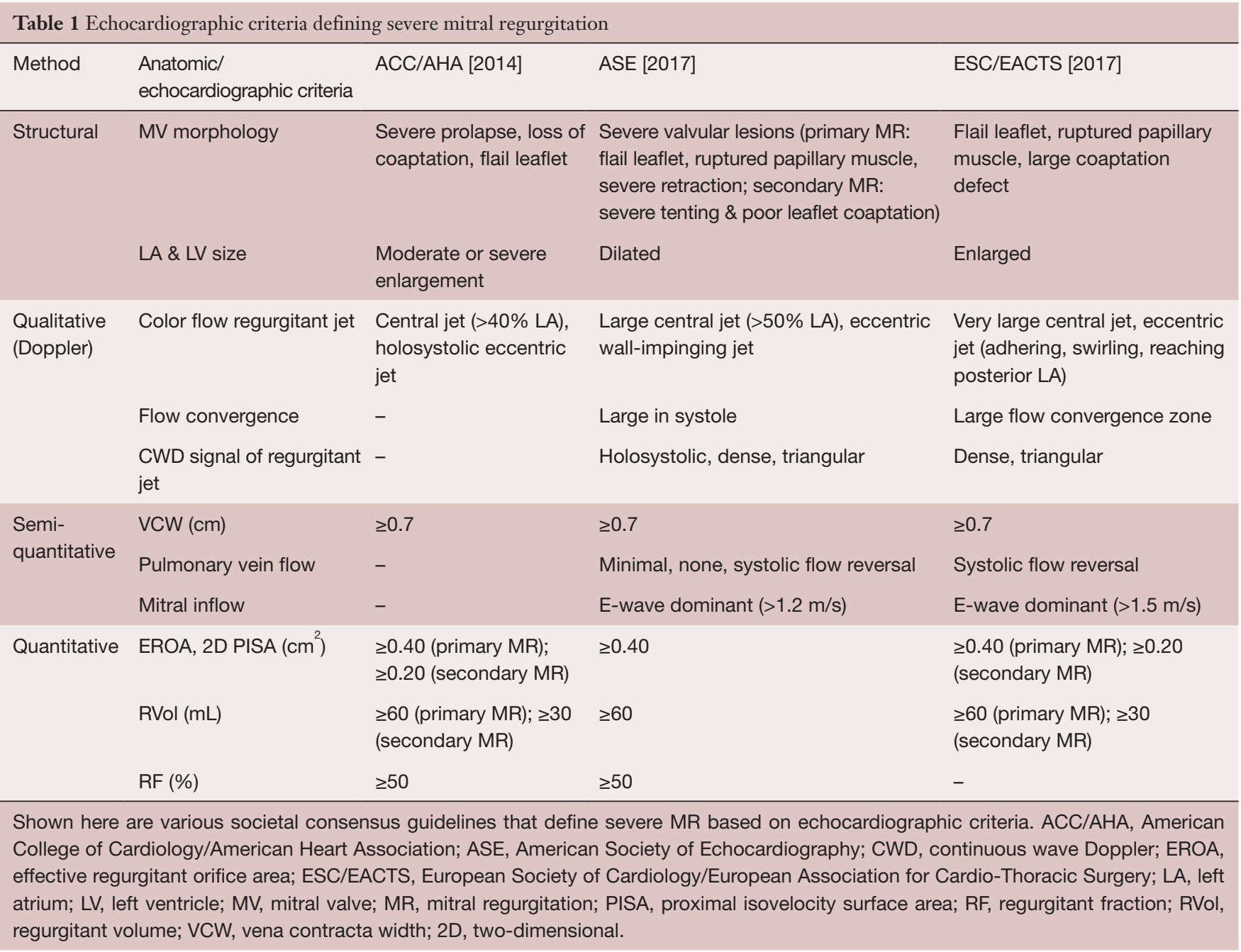

alternative to MitraClip for percutaneous edge-to-edge repair if the patient's anatomy allows. The device has received CE mark approval in Europe and is currently being evaluated in clinical trials in the United States (22). The echocardiographic indications for its use are similar to that of the MitraClip system, as are the screening and procedural planning considerations described above (23).

\section{NeoChord \& Harpoon}

Both the NeoChord and Harpoon repair systems implant neo-chordae in the beating heart to correct regurgitation secondary to degenerative disease and require accurate preprocedural echocardiographic assessment of MR physiology and MV anatomy (24,25). Here, treatment of isolated P2 prolapse is most likely to have successful outcomes. Furthermore, these devices may not be efficacious in patients with short posterior leaflets relative to the anteroposterior mitral annular dimensions, since rectification of the prolapse does not ensure sufficient coaptation between the two leaflets (4). TTE and computed tomography (CT) are also often useful in evaluating the optimal intercostal space and location for the minithoracotomy required for eventual transapical access.

\section{TMVI \& the mitral annulus}

Devices that target the MV annulus include the Cardioband (Edwards Lifesciences Inc., Irvine, CA) and Carillon (Cardiac Dimensions, Kirkland, WA) systems.

\section{Cardioband}

Mimicking surgical annuloplasty, the Cardioband system 

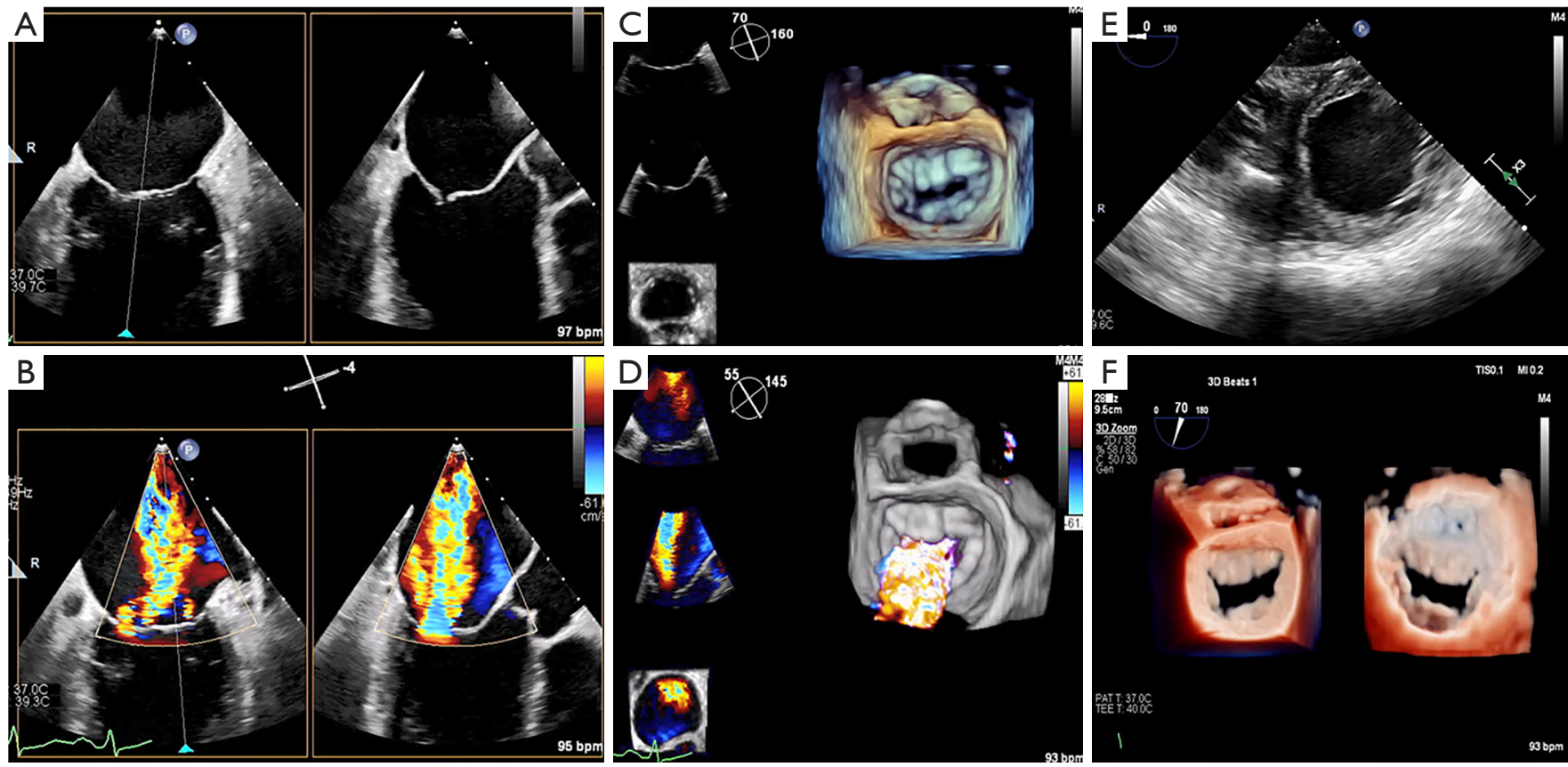

Figure 6 TEE \& procedural planning for MitraClip. TEE is crucial for anatomic evaluation and procedural planning for percutaneous edge-to-edge mitral repair. (A,B) The biplane commissural and $\mathrm{X}$-plane-to-LVOT views assess the mitral valve across all segments and is useful for analyzing valvular anatomy at the target grasping location. (C,D) The 3D en face (surgeon's) view here affirms the presence of severe functional MR and failure of leaflet coaptation. (E) The short-axis view shows a severely dilated and dysfunctional left ventricle. (F) Using the transillumination tool on the Philips TEE software, the 3D en face Truview enables simultaneous, high-resolution assessments of pathoanatomy from both the atrial (left) and ventricular (right) views of the mitral valve. LVOT, left ventricular outflow tract; MR, mitral regurgitation; TEE, transesophageal echocardiography; 3D, three-dimensional.

consists of a series of metal anchors within a Dacron band that are circumferentially implanted in a step-wise fashion around the MV annulus (26). Preprocedural evaluation mandates 2D and 3D TEE and MDCT for all patients. The former is used to assess MR etiology and severity as previously described, and the latter is required for anatomic planning. Specifically, the posterior mitral annular perimeter should be measured using a cubic-spline interpolation measurement from a semi-automated workflow, following which the implant length and number of required anchors is determined. Next, to assess tissue adequacy for anchoring, the width of the annular shelf should be evaluated around the planned implantation circumference (acceptable parameters include mid-anchor-to-left ventricle $>4 \mathrm{~mm}$ and anchor head-to-leaflet hinge $<8 \mathrm{~mm}$ ). The location of the circumflex coronary artery and coronary sinus should also be noted given their proximity to the mitral annulus (anchor-to-circumflex coronary $>2.5 \mathrm{~mm}$ is ideal). Here, TEE can also be used to evaluate the position of the artery at the level of the anterolateral trigone. Finally, MDCT can be used to optimize the location of the transseptal puncture $(4,15)$.

\section{Carillon mitral contour system}

The Carillon device reduces MR by cinching the annulus, reducing the anteroposterior annular diameter and improving leaflet coaptation. It makes use of two selfexpandable nitinol anchors of various diameters, connected by a curvilinear segment of various lengths, and is delivered via the coronary sinus. Recently, the REDUCE FMR trial, a blinded, randomized, proof-of-concept, sham-controlled study that evaluated 120 patients with symptomatic functional MR, reported that use of this device significantly reduced regurgitant and $\mathrm{LV}$ volumes at one year (27). Currently, only those with secondary MR are eligible candidates for the Carillon system, with a pivotal trial underway in the United States; the presence of primary MR or MAC are contraindications.

Patient selection for implantation of this system requires preprocedural measurements of the coronary sinus and 

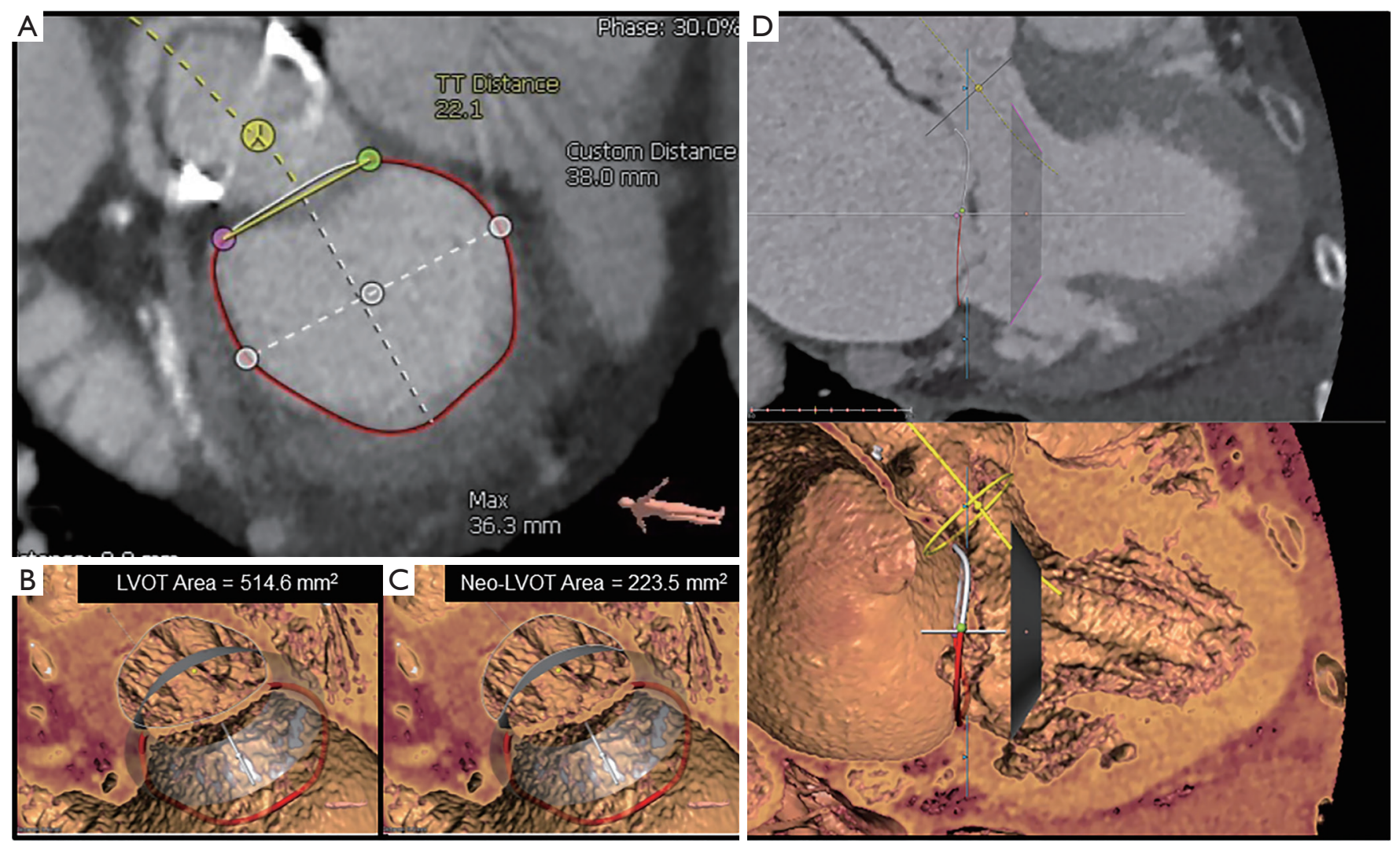

Figure 7 MDCT \& procedural planning for TMVR. Preprocedural MDCT analysis for TMVR with the Intrepid system (Medtronic, Minneapolis, MN). (A) Assessment of the mitral annular plane at end-systole yields a perimeter of 119 mm, with an equivalent diameter of $37.2 \mathrm{~mm}$. This would fit a $42 \mathrm{~mm}$ device. (B,C) Measurements of the LVOT and neo-LVOT suggest a low risk of LVOT obstruction. (D) The Intrepid TMVR device (trapezoid) is virtually placed at the native annulus to model and simulate its fit within the left ventricle and assess LVOT clearance. LVOT, left ventricular outflow tract; MDCT, multidetector computed tomography; TMVR, transcatheter mitral valve replacement.

great cardiac vein using invasive venography. Specifically, to accommodate $\mathrm{a} \geq 60 \mathrm{~mm}$ device and pull $\geq 3 \mathrm{~cm}$ of tension to cinch the annulus, the vein should ideally be $\geq 9-10 \mathrm{~mm}$. CT has yet to be validated as a viable alternative to venography for assessing venous dimensions (28).

\section{Transcatheter MV replacement}

As with the various TMVI therapies described, successful TMVR relies on multimodality imaging for patient selection, procedural guidance and post-procedural assessment. In particular, preprocedural evaluation relies heavily on a comprehensive MDCT analysis that allows measurements of MV dimensions and geometry, along with anatomic assessment of the structures surrounding the landing zone of the TMVR device (Figure 7). Relevant annular dimensions include the intercommissural and septolateral diameters, area, perimeter, displacement and tenting area and volume (3). Annular sizing is performed at both end-systole and end-diastole.

CT-based measurement requirements also vary by device specifications. For instance, assessment of chordal and leaflet anatomy is imperative for TMVR devices that anchor via leaflet grasping. On the other hand, to gauge the risk of obstruction during positioning of valves that anchor behind the leaflets or trigones, evaluation of the distance to the papillary muscles may be important (4). Furthermore, to predict the risk of LVOTO, some authors have suggested MDCT-based reconstruction and creation of a virtual $\mathrm{D}$-shaped annulus defined by the inter-trigonal distance $(29,30)$. Finally, the presence of MAC should be noted and characterized as this may impede proper anchoring (31). 

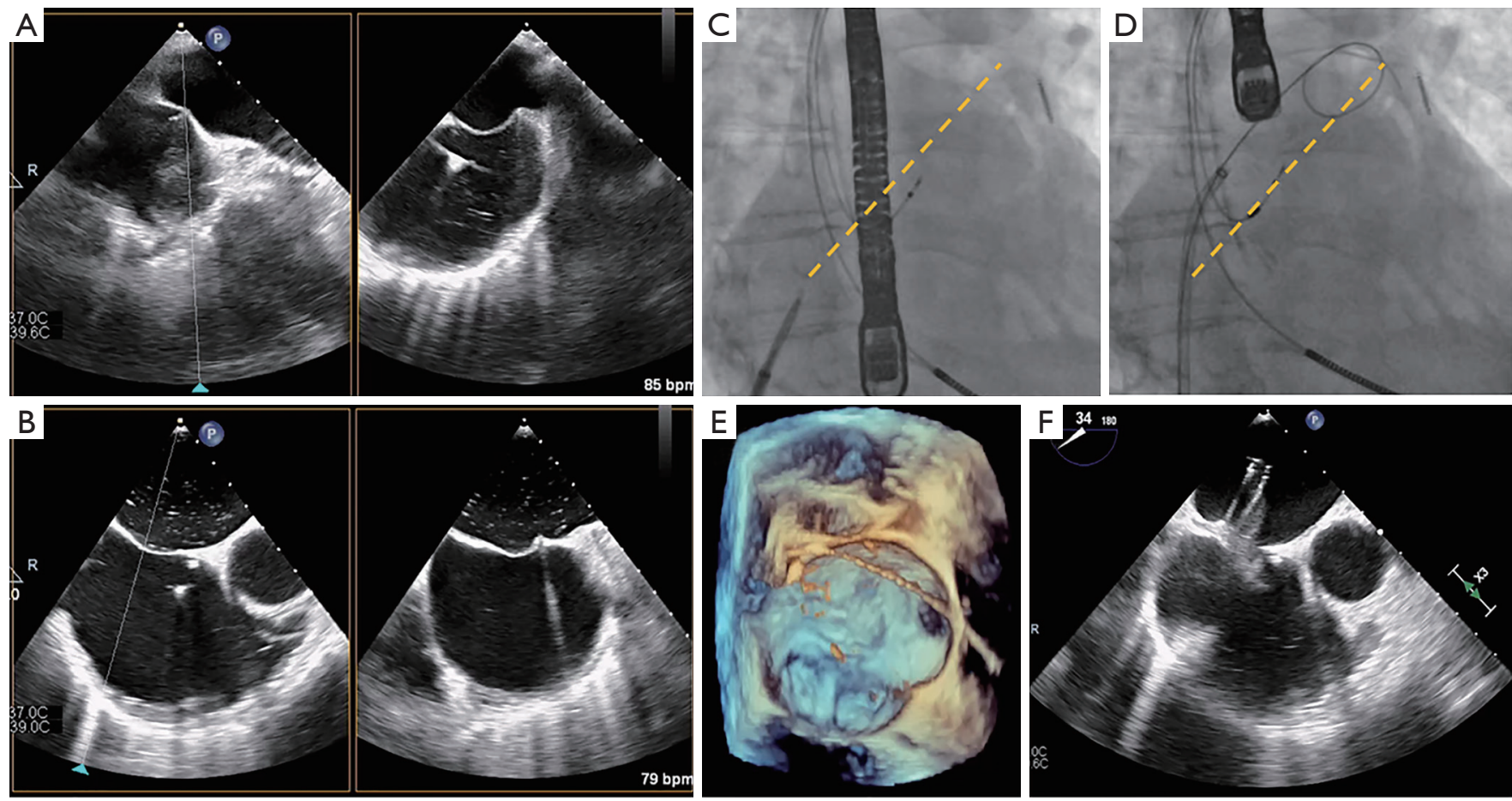

Figure 8 Transseptal puncture in MitraClip. Transseptal puncture in MitraClip is guided by TEE and fluoroscopy. (A,B) Bi-plane TEE imaging permits simultaneous visualization of the bicaval and four-chamber views for puncture localization, demonstrated by the septal tenting here (usually performed in a mid-posterior location within the interatrial septum). (C,D) Fluoroscopy is used to estimate the distance between the puncture site and left atrial appendage (yellow dotted line), along with the projected trajectory of the device as evidenced by the stiff wire pathway, to facilitate subsequent SGC/CDS steering. (E,F) 3D imaging and the aortic-valve short-axis view here confirm the wire and SGC guide trajectory in the left atrium. CDS, clip delivery system; SGC, steerable guide catheter; TEE, transesophageal echocardiography; 3D, three-dimensional.

\section{Intra-procedural imaging guidance}

Intraoperative imaging guidance for virtually all TMVI procedures is predicated upon $2 \mathrm{D}$ and $3 \mathrm{D}$ TEE and fluoroscopy. Several of these are discussed below.

\section{MitraClip}

The initial, and arguably the most important, step prior to MitraClip placement is the transseptal puncture. This is usually performed in a mid-posterior location within the inter-atrial septum, approximately $4.0-4.5 \mathrm{~cm}$ basal to the annular plane. For puncture localization, bi-plane TEE imaging allows concomitant visualization of the bicaval and four-chamber views. This may also be facilitated by the use of fusion imaging systems that overlay TEE images onto fluoroscopy (32). 3D imaging of needle tenting at the inter-atrial septum then confirms the puncture trajectory
(Figure 8) $(33,34)$. Device extrusion from the delivery sheath should then be visualized to avoid contact injury with the Coumadin ridge, left atrial appendage or left atrial wall. The clip delivery system is straddled across the commissures and the clip is opened under TEE-3D visualization to ensure its proper orientation perpendicular to the commissures. Following confirmation of clip position in the bi-plane commissural X-plane-to-LVOT views, the device is passed into the left ventricle. Bi-plane color Doppler imaging is then used to confirm clip placement at the MR jet location, and the clip is pulled back toward the leaflets. Live color Doppler imaging may be used to confirm MR reduction as the clip arms are closed. After leaflet grasping, TEE-3D shows a tissue bridge that confirms optimal leaflet grasping at a suitable location. Improvements in pulmonary vein flow are indicative of significant MR reduction. At this point, transmitral CWD may be used to assess any increase in 

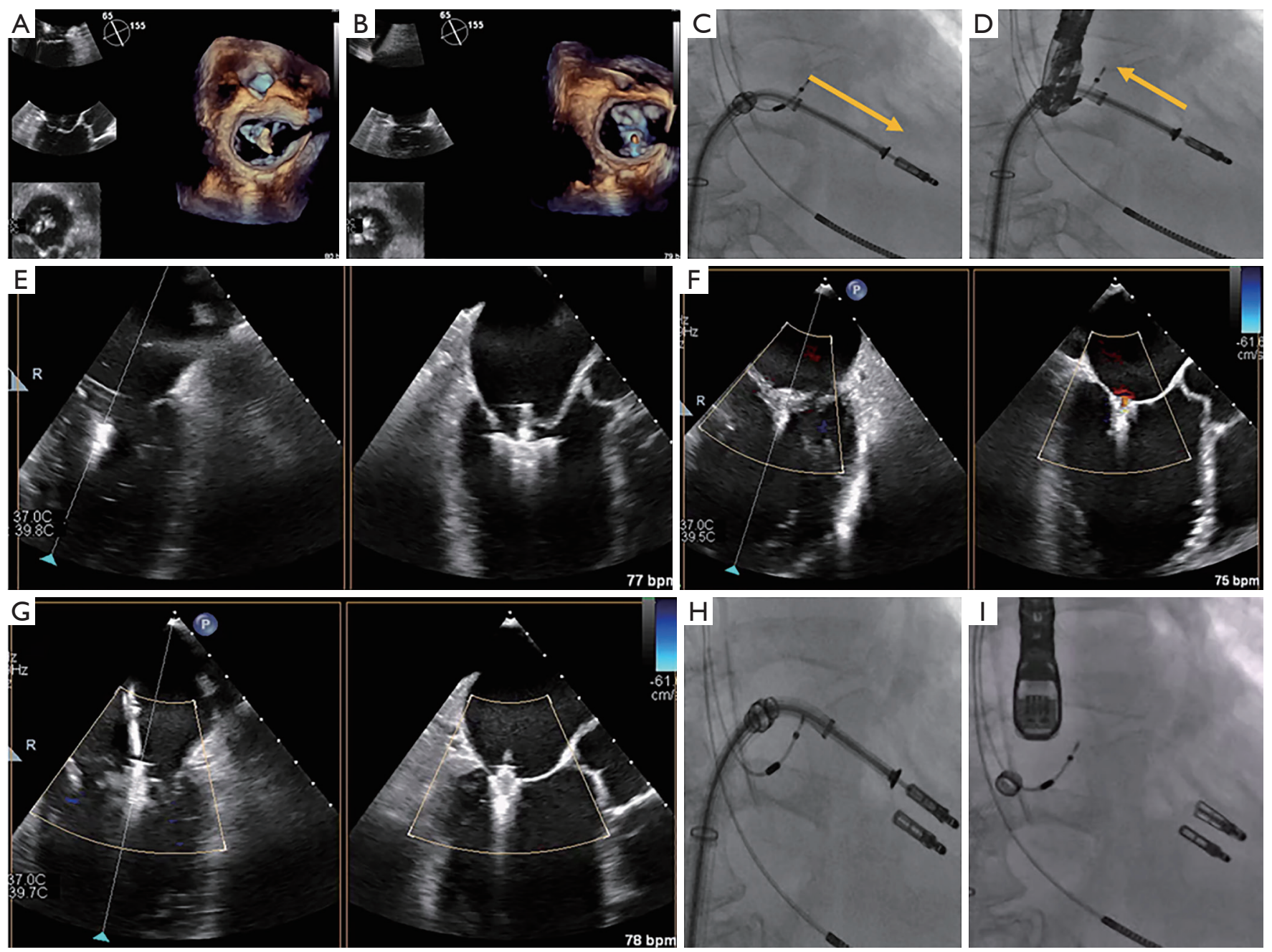

Figure 9 TEE- \& fluoroscopy-based guidance of percutaneous edge-to-edge repair. Use of the MitraClip XTR system in a patient with severe functional MR is shown here. Device orientation is visualized using the $3 \mathrm{D}$ en face view with the Clip in the (A) left atrium and (B) left ventricle. (C,D) Proper alignment is then confirmed on fluoroscopy (orange arrows), showing no rotation of the Clip as it is advanced into the left ventricle and retracted during leaflet grasping. (E) The leaflets are grasped by the MitraClip XTR system and (F) the first of two XTR clips is deployed. (G) The second XTR clip is positioned lateral to the previous one, and (H,I) MitraClip alignment is once again ascertained on fluoroscopy. TEE, transesophageal echocardiography; $\mathrm{MR}$, mitral regurgitation; 3D, three-dimensional.

gradients, which can be rectified by clip repositioning and regrasping (Figure 9) (15).

Post-implant assessment is crucial following percutaneous edge-to-edge repair (35). Multiplanar 3D reconstruction permits planimetry of each mitral orifice; areas are then added to yield the total MVA following clip placement. Post-clip MR can be assessed using 2D color Doppler imaging and 3D color Doppler direct planimetry of the VC areas (Figure 10). A recent study by Avenatti et al. showed that post-procedural VC area $<0.27 \mathrm{~cm}^{2}$ was associated with clinical improvements in New York Heart Association functional class at 30-day follow-up (36). Finally, complications such as pericardial effusion, clip detachment and leaflet damage should be assessed via TEE.

\section{PASCAL, NeoChord, \& Harpoon}

Procedural guidance of the PASCAL system is similar to that described above. However, both the NeoChord and Harpoon systems differ in that they access the left ventricle 

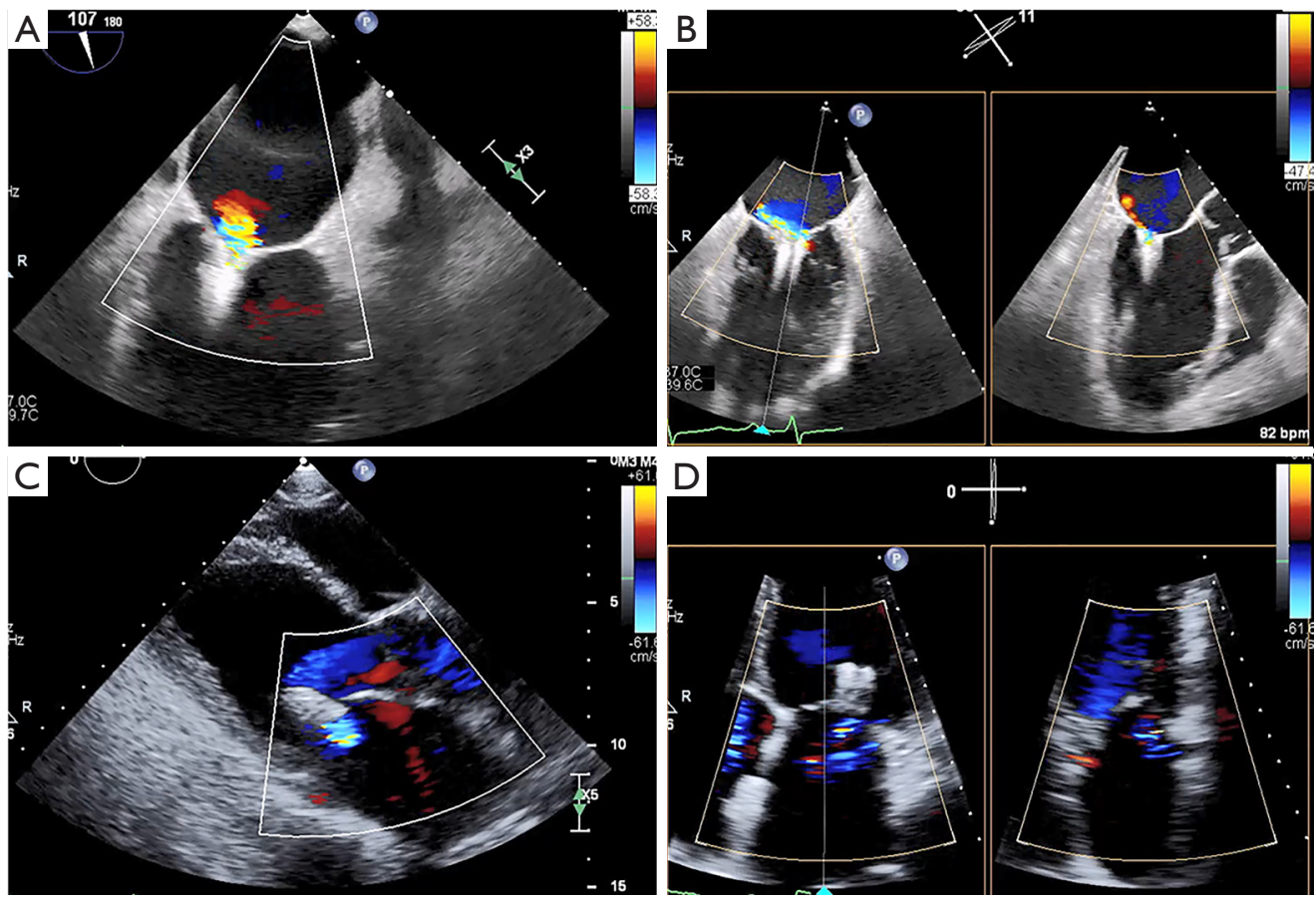

Figure 10 Post-procedural assessment following MitraClip for severe functional MR. Echocardiography is essential for valvular assessment following a MitraClip procedure for severe functional MR. (A,B) TEE shows proper clip positioning with substantial reductions in the severity of MR. (C,D) Pre-discharge TTE shows mild MR with an ejection fraction of approximately $25 \%$. MR, mitral regurgitation; TEE, transesophageal echocardiography; TTE, transthoracic echocardiography.

transapically. Here, TTE is often used to determine the ideal intercostal space and location for mini-thoracotomy. CT, which enables visualization of the device trajectory, may also be used to guide transapical access of both the DS 1000 and the Harpoon systems (4,24). Following this, device insertion, leaflet grasping and tensioning are guided by TEE using the bi-commissural, X-plane-to-LVOT and 3D en face views of the MV.

\section{Cardioband}

TEE-2D, -3D and fluoroscopy are essential for procedural guidance of the Cardioband system. This technology relies on transseptal access and MDCT is often used to determine the optimal location for puncture (ideally $3.0-4.5 \mathrm{~cm}$ above the annular plane). In addition, MDCT can recreate the fluoroscopic views used for device implantation. For instance, the short-axis view with the MV en face view is used to set the first anchor anterior to the lateral commissure near the trigone, while the two- and threechamber views are used to implant the medial anchors (4). Each anchor is sequentially deployed from the first location posteriorly until one reaches the posteromedial commissure. During implantation, anchor insertion within the annulus can be confirmed with multiplane $3 \mathrm{D}$ imaging. Once completed, TEE-3D is used to evaluate the mitral annulus and assess the severity of MR via multiplanar reconstruction of annular dimensions and $\mathrm{MV}$ and regurgitant orifice areas $(15,37)$.

\section{Transcatheter MV replacement}

Fluoroscopy with a co-planar view of the mitral annulus is the mainstay of imaging-based guidance of TMVR (38). Preprocedural MDCT-based optimization of fluoroscopic angulations is frequently used to achieve the co-planar view. In addition, MDCT can also facilitate use of the coronary sinus guidewire, which is often used as a landmark that identifies the annular plane on fluoroscopy. For device implantation, both the bi-commissural and X-plane-toLVOT views are used to navigate device insertion, valve positioning and deployment, while fluoroscopy is used to aid TEE in guiding device insertion and positioning, valve deployment and delivery system retrieval (Figure 11) $(39,40)$. 

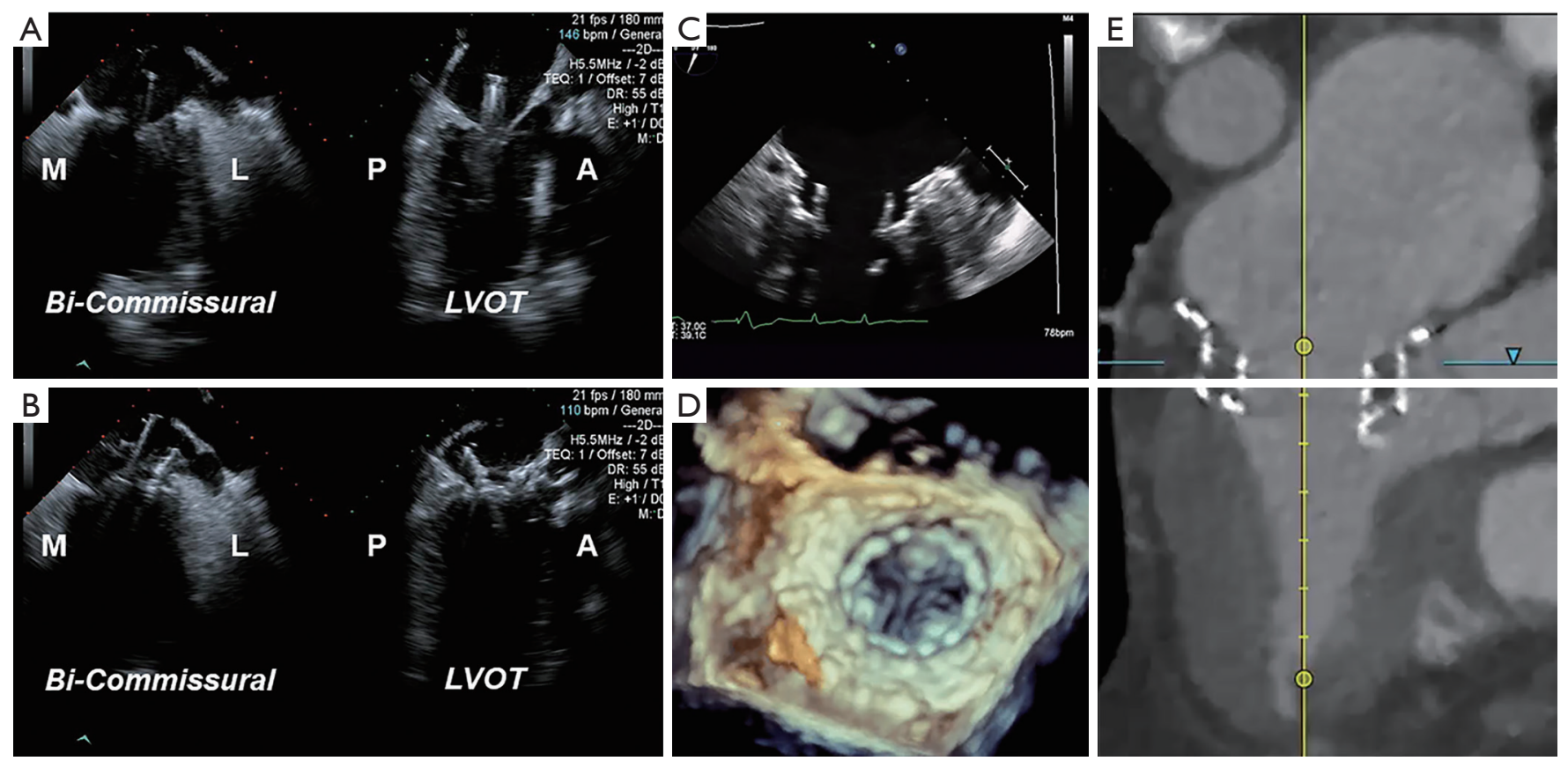

Figure 11 Intraprocedural imaging-based guidance of TMVR. Imaging-based guidance of TMVR with the Medtronic Intrepid system is shown here. $(\mathrm{A}, \mathrm{B})$ The bi-commissural and X-plane-to-LVOT views on TEE are used to guide advancement of the device delivery system into the left atrium. Valve positioning and deployment follow. (C,D) Post-procedural TEE shows a well-seated Intrepid TMVR device. (E) MDCT assessment shows the valve in the appropriate position with no LVOTO. A, anterior; M, medial; MDCT, multidetector computed tomography; L, lateral; LVOT, left ventricular outflow tract; LVOTO, left ventricular outflow tract obstruction; P, posterior; TEE, transesophageal echocardiography; TMVR, transcatheter mitral valve replacement.

\section{Conclusions}

With the evolving landscape of TMVI, the paradigm of treating patients with severe MR is changing. As evidenced in this article, this burgeoning field of transcatheter mitral therapies relies heavily on multi-modality cardiac imaging. Indeed, it is imperative that the interventional surgeon of the future be well versed in the roles of echocardiography, MDCT, fluoroscopy and most recently, CMR, in order to optimize preprocedural patient selection, procedural planning, intra-procedural imaging-based guidance and post-procedural assessment of MV morphology and function. As outcomes of TMVI improve, cardiac imaging will undoubtedly continue to play an essential role in the success of percutaneous MV repair and replacement.

\section{Acknowledgments}

Funding: None.

\section{Footnote}

Conflicts of Interest: Dr. GHLT is a consultant for Medtronic, Abbott Structural Heart, and W. L. Gore \& Associates. Dr. RPM reports the following financial interests/ arrangements or affiliations: Steering Committee, Abbott TRILUMINATE Pivotal; Speakers Bureau, Edwards Lifesciences; Speakers Bureau, Medtronic Heart Valves; Medical Advisory Board, CardioCare-Edwards; Executive Committee, Medtronic TMVR, Officer and Stock Holder, Bay Labs-AI Company. The other authors have no conflicts of interest to declare.

Open Access Statement: This is an Open Access article distributed in accordance with the Creative Commons Attribution-NonCommercial-NoDerivs 4.0 International License (CC BY-NC-ND 4.0), which permits the noncommercial replication and distribution of the article with the strict proviso that no changes or edits are made and the 
original work is properly cited (including links to both the formal publication through the relevant DOI and the license). See: https://creativecommons.org/licenses/by-nc-nd/4.0/.

\section{References}

1. Nkomo VT, Gardin JM, Skelton TN, et al. Burden of valvular heart diseases: a population-based study. Lancet 2006;368:1005-11.

2. Bonow RO, O'Gara PT, Adams DH, et al. 2020 Focused Update of the 2017 ACC Expert Consensus Decision Pathway on the Management of Mitral Regurgitation: A Report of the American College of Cardiology Solution Set Oversight Committee. J Am Coll Cardiol 2020;75:2236-70.

3. Blanke P, Naoum C, Webb J, et al. Multimodality Imaging in the Context of Transcatheter Mitral Valve Replacement: Establishing Consensus Among Modalities and Disciplines. JACC Cardiovasc Imaging 2015;8:1191-208.

4. Bax JJ, Debonnaire P, Lancellotti P, et al. Transcatheter Interventions for Mitral Regurgitation: Multimodality Imaging for Patient Selection and Procedural Guidance. JACC Cardiovasc Imaging 2019;12:2029-48.

5. Baumgartner H, Falk V, Bax JJ, et al. 2017 ESC/EACTS Guidelines for the management of valvular heart disease. Eur Heart J 2017;38:2739-91.

6. Nishimura RA, Otto CM, Bonow RO, et al. 2014 AHA/ ACC guideline for the management of patients with valvular heart disease: a report of the American College of Cardiology/American Heart Association Task Force on Practice Guidelines. J Am Coll Cardiol 2014;63:e57-185.

7. Carpentier A. Cardiac valve surgery--the "French correction". J Thorac Cardiovasc Surg 1983;86:323-37.

8. Pepi M, Tamborini G, Maltagliati A, et al. Headto-head comparison of two- and three-dimensional transthoracic and transesophageal echocardiography in the localization of mitral valve prolapse. J Am Coll Cardiol 2006;48:2524-30.

9. Van Mieghem NM, Piazza N, Anderson RH, et al. Anatomy of the mitral valvular complex and its implications for transcatheter interventions for mitral regurgitation. J Am Coll Cardiol 2010;56:617-26.

10. Zoghbi WA, Adams D, Bonow RO, et al.

Recommendations for Noninvasive Evaluation of Native Valvular Regurgitation: A Report from the American Society of Echocardiography Developed in Collaboration with the Society for Cardiovascular Magnetic Resonance. J Am Soc Echocardiogr 2017;30:303-71.
11. Tops LF, Van de Veire NR, Schuijf JD, et al. Noninvasive evaluation of coronary sinus anatomy and its relation to the mitral valve annulus: implications for percutaneous mitral annuloplasty. Circulation 2007;115:1426-32.

12. Blanke P, Naoum C, Dvir D, et al. Predicting LVOT Obstruction in Transcatheter Mitral Valve Implantation: Concept of the Neo-LVOT. JACC Cardiovasc Imaging 2017;10:482-5.

13. Yoon SH, Bleiziffer S, Latib A, et al. Predictors of Left Ventricular Outflow Tract Obstruction After Transcatheter Mitral Valve Replacement. JACC Cardiovasc Interv 2019;12:182-93.

14. Lancellotti P, Moura L, Pierard LA, et al. European Association of Echocardiography recommendations for the assessment of valvular regurgitation. Part 2: mitral and tricuspid regurgitation (native valve disease). Eur J Echocardiogr 2010;11:307-32.

15. Khalique OK, Hahn RT. Percutaneous Mitral Valve Repair: Multi-Modality Cardiac Imaging for Patient Selection and Intra-Procedural Guidance. Front Cardiovasc Med 2019;6:142.

16. Uretsky S, Argulian E, Narula J, et al. Use of Cardiac Magnetic Resonance Imaging in Assessing Mitral Regurgitation: Current Evidence. J Am Coll Cardiol 2018;71:547-63.

17. Lopez-Mattei JC, Ibrahim H, Shaikh KA, et al. Comparative Assessment of Mitral Regurgitation Severity by Transthoracic Echocardiography and Cardiac Magnetic Resonance Using an Integrative and Quantitative Approach. Am J Cardiol 2016;117:264-70.

18. Feldman T, Kar S, Rinaldi M, et al. Percutaneous mitral repair with the MitraClip system: safety and midterm durability in the initial EVEREST (Endovascular Valve Edge-to-Edge REpair Study) cohort. J Am Coll Cardiol 2009;54:686-94.

19. Hahn RT. Transcathether Valve Replacement and Valve Repair: Review of Procedures and Intraprocedural Echocardiographic Imaging. Circ Res 2016;119:341-56.

20. Altiok E, Hamada S, Brehmer K, et al. Analysis of procedural effects of percutaneous edge-to-edge mitral valve repair by $2 \mathrm{D}$ and $3 \mathrm{D}$ echocardiography. Circ Cardiovasc Imaging 2012;5:748-55.

21. Weinmann K, Markovic S, Rottbauer W, et al. First experience with the MitraClip XTR device for extensive mitral valve prolapse (Barlow's disease). EuroIntervention 2018;14:e1276-7.

22. Praz F, Spargias K, Chrissoheris M, et al. Compassionate use of the PASCAL transcatheter mitral valve repair 
system for patients with severe mitral regurgitation: a multicentre, prospective, observational, first-in-man study. Lancet 2017;390:773-80.

23. Praz F, Windecker S, Kapadia S. PASCAL: A New Addition to the Armamentarium of Transcatheter Repair Systems for Mitral Leaflet Approximation. JACC Cardiovasc Interv 2019;12:1379-81.

24. Colli A, Manzan E, Fabio FZ, et al. TEE-guided transapical beating-heart neochord implantation in mitral regurgitation. JACC Cardiovasc Imaging 2014;7:322-3.

25. Gammie JS, Wilson P, Bartus K, et al. Transapical Beating-Heart Mitral Valve Repair With an Expanded Polytetrafluoroethylene Cordal Implantation Device: Initial Clinical Experience. Circulation 2016;134:189-97.

26. Messika-Zeitoun D, Nickenig G, Latib A, et al. Transcatheter mitral valve repair for functional mitral regurgitation using the Cardioband system: 1 year outcomes. Eur Heart J 2019;40:466-72.

27. Witte KK, Lipiecki J, Siminiak T, et al. The REDUCE FMR Trial: A Randomized Sham-Controlled Study of Percutaneous Mitral Annuloplasty in Functional Mitral Regurgitation. JACC Heart Fail 2019;7:945-55.

28. Schofer J, Siminiak T, Haude M, et al. Percutaneous mitral annuloplasty for functional mitral regurgitation: results of the CARILLON Mitral Annuloplasty Device European Union Study. Circulation 2009;120:326-33.

29. Blanke P, Dvir D, Cheung A, et al. A simplified D-shaped model of the mitral annulus to facilitate CT-based sizing before transcatheter mitral valve implantation. J Cardiovasc Comput Tomogr 2014;8:459-67.

30. Naoum C, Leipsic J, Cheung A, et al. Mitral Annular Dimensions and Geometry in Patients With Functional Mitral Regurgitation and Mitral Valve Prolapse: Implications for Transcatheter Mitral Valve Implantation. JACC Cardiovasc Imaging 2016;9:269-80.

31. Guerrero M, Urena M, Himbert D, et al. 1-Year Outcomes of Transcatheter Mitral Valve Replacement in Patients With Severe Mitral Annular Calcification. J Am Coll Cardiol 2018;71:1841-53.

32. Faletra FF, Biasco L, Pedrazzini G, et al. Echocardiographic-Fluoroscopic Fusion Imaging in

Cite this article as: Sengupta A, Alexis SL, Zaid S, Tang GHL, Lerakis S, Martin RP. Imaging the mitral valve: a primer for the interventional surgeon. Ann Cardiothorac Surg 2021;10(1): 28-42. doi: 10.21037/acs-2020-mv-16
Transseptal Puncture: A New Technology for an Old Procedure. J Am Soc Echocardiogr 2017;30:886-95.

33. Faletra FF, Nucifora G, Ho SY. Imaging the atrial septum using real-time three-dimensional transesophageal echocardiography: technical tips, normal anatomy, and its role in transseptal puncture. J Am Soc Echocardiogr 2011;24:593-9.

34. Altiok E, Becker M, Hamada S, et al. Optimized guidance of percutaneous edge-to edge repair of the mitral valve using real-time 3-D transesophageal echocardiography. Clin Res Cardiol 2011;100:675-81.

35. Zoghbi WA, Asch FM, Bruce C, et al. Guidelines for the Evaluation of Valvular Regurgitation After Percutaneous Valve Repair or Replacement: A Report from the American Society of Echocardiography Developed in Collaboration with the Society for Cardiovascular Angiography and Interventions, Japanese Society of Echocardiography, and Society for Cardiovascular Magnetic Resonance. J Am Soc Echocardiogr 2019;32:431-75.

36. Avenatti E, Mackensen GB, El-Tallawi KC, et al. Diagnostic Value of 3-Dimensional Vena Contracta Area for the Quantification of Residual Mitral Regurgitation After MitraClip Procedure. JACC Cardiovasc Interv 2019;12:582-91.

37. Pozzoli A, Zuber M, Taramasso M, et al. 3D echofluoro fusion imaging to guide Cardioband transcatheter mitral annuloplasty. Eur Heart J Cardiovasc Imaging 2018;19:827.

38. El Hajj SC, Eleid MF. Transcatheter Mitral Valve Replacement: An Update on the Current Literature. Curr Treat Options Cardiovasc Med 2019;21:35.

39. Blanke P, Dvir D, Naoum C, et al. Prediction of fluoroscopic angulation and coronary sinus location by CT in the context of transcatheter mitral valve implantation. J Cardiovasc Comput Tomogr 2015;9:183-92.

40. Theriault-Lauzier P, Andalib A, Martucci G, et al. Fluoroscopic anatomy of left-sided heart structures for transcatheter interventions: insight from multislice computed tomography. JACC Cardiovasc Interv 2014;7:947-57. 